\title{
Uma solução particular baseada em funções de Green para formulações nodais derivadas da equação de transporte bidimensional
}

\author{
Anderson Tres, Camila B. Picoloto, \\ Programa de Pós-Graduação em Matemática Aplicada, UFRGS, \\ Av. Bento Gonçalves 9500 - 91509-900, Porto Alegre, RS, \\ Programa de Pós-Graduação em Engenharia Mecânica, UFRGS, \\ Rua Sarmento Leite, 425 - 90050-170, Porto Alegre, RS \\ E-mail: andersontres.mat@gmail.com, camila_becker_87@hotmail.com, \\ Liliane B. Barichello Rudnei D. da Cunha, \\ Instituto de Matemática, UFRGS, \\ Av. Bento Gonçalves 9500 - 91509-900, Porto Alegre, RS \\ E-mail: lbaric@mat.ufrgs.br, rcunha@mat.ufrgs.br.
}

Resumo: Neste trabalho, investiga-se um problema de transporte de partículas definido em um dominio retangular com uma fonte de nêutrons isotrópica, cercado por condições de contorno de vácuo em geometria cartesiana bidimensional. A abordagem proposta, já utilizada anteriormente, reduz a complexidade do modelo bidimensional através de esquemas nodais, juntamente com a aplicação do método de ordenadas discretas analítico (ADO). O esquema nodal é definido em todo o domínio do problema e como usualmente em métodos nodais, relações entre os fluxos médios e os fluxos desconhecidos nos contornos, são introduzidos. O método ADO é usado para desenvolver soluções para as equações integradas unidimensionais, que são explícitas em termos das variáveis espaciais. A técnica utilizada conduz a uma redução da ordem dos sistemas de autovalores associados, proporcionando desse modo, soluções de forma mais eficientes no ponto de vista computacional. Para o caso teste aqui analisado, diferentemente de trabalhos anteriores, soluçôes particulares mais gerais são utilizadas baseadas em funções de Green.

Palavras-chave: Método de ordenadas discretas analítico, equação de transporte bidimensional, funções de Green

\section{Introdução}

A solução de problemas de transporte de nêutrons multidimensionais tem sido um tópico de pesquisa de grande interesse, devido ao amplo campo de aplicações [1,12]. Neste contexto, daremos atenção aos métodos nodais $[2,3,7]$, já que estes são comumente utilizados na resolução de problemas multidimensionais onde, através da integração em uma das variáveis espaciais, reduz-se o sistema de EDP's (originados da discretização da integral angular) em sistemas de EDO's. O uso de esquemas nodais reduz a complexidade do modelo e possibilita a utilização de vários instrumentos de análise espacial $[5,6,8,11]$.

O método ADO [4] tem sido intensivamente utilizado com sucesso para encontrar a solução das equações nodais em ordenadas discretas, derivadas do problema de transporte de nêutrons bidimensional, de uma maneira concisa e exata, a baixo custo computacional $[5,6,10]$. O problema teste apresentado aqui é diferente daquele tratado em $[5,6]$. O mesmo foi abordado, no entanto, na referência [10], sendo que aqui, a solução particular é derivada através das funções de Green, que se torna mais geral que o caso tratado em [10], baseado na proposição de soluções 
particulares constantes. Desta maneira, busca-se estender a investigação que vem sendo feita com o método ADO na solução de problemas bidimensionais [5,6,10], objetivando abordar uma variedade de problemas testes, no sentido de obter uma análise ampla do desempenho da formulação proposta e validar os resultados numéricos obtidos a partir de formulações teóricas diferenciadas.

Na próxima seção, a equação de transporte de nêutrons bidimensional em ordenadas discretas é aplicada a um problema de fonte fixa em meios não-multiplicativos. A equação é integrada na variável $y$ de modo a fornecer um sistema de EDO's, onde os termos desconhecidos que aparecem nos contornos são aproximados, de maneira que o problema unidimensional na variável $x$ fique independente da variável $y$. Na seção 3 o Método ADO é aplicado ao problema unidimensional na variável $x$ onde, foi possível reduzir à metade, a ordem do problema de autovalores. As soluções do problema homogêneo são então definidas. Na seção 3.1, a solução particular via funções de Green é proposta. As considerações finais deste trabalho estão na seção 4.

\section{Formulação do Problema}

Considera-se o problema de transporte definido numa região retangular $R$, com $x \in[0, a] \mathrm{e}$ $y \in[0, b]$ centrado na origem de um sistema cartesiano. Na região definida em $\left[0, a_{s}\right] \times\left[0, b_{s}\right]$ dentro de $R$, existe uma fonte isotrópica de nêutrons, de acordo com Loyalka e Tsai [9].

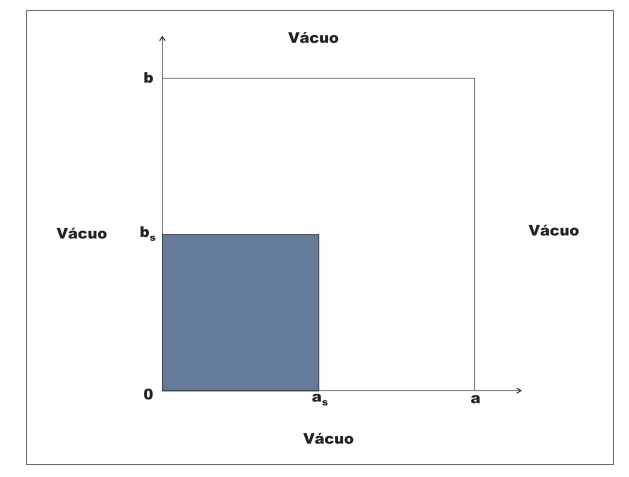

Figura 1: Domínio R.

Considerando a equação para o fluxo angular de nêutrons, em meio não-multiplicativo, homogêneo, com fonte isotrópica fixa, escrita em ordenadas discretas

$$
\mu_{m} \frac{\partial}{\partial x} \Psi\left(x, y, \boldsymbol{\Omega}_{m}\right)+\eta_{m} \frac{\partial}{\partial y} \Psi\left(x, y, \boldsymbol{\Omega}_{m}\right)+\sigma_{t} \Psi\left(x, y, \boldsymbol{\Omega}_{m}\right)=Q(x, y)+\frac{\sigma_{s}}{4} \sum_{k=1}^{M} w_{k} \Psi\left(x, y, \boldsymbol{\Omega}_{k}\right)
$$

para $m=1, \ldots, M \operatorname{com} M=N(N+2) / 2$, onde $w_{m}$ são os pesos asociados as direções $\boldsymbol{\Omega}_{m}=$ $\left(\mu_{m}, \eta_{m}\right), \sigma_{t}$ e $\sigma_{s}$ são as seções de choque total e de espalhamento, respectivamente, e $Q(x, y)$ é o termo fonte de nêutrons.

Objetivando analisar a equação para a direção $x$, optou-se por ordenar o conjunto de direções $\left(\omega_{m}, \boldsymbol{\Omega}_{m}\right)$ definidas por $\mu_{m}>0$ aos índices $m=1, \ldots, M / 2$ e $\mu_{m}<0$ aos índices $m=M / 2+1, \ldots, M$. Desta forma, integrando a eq. (1) em todo $y$, obtemos as equações nodais unidimensionais na variável $x$ como

$$
\begin{aligned}
& \mu_{m} \frac{d}{d x} \Psi_{y}\left(x, \boldsymbol{\Omega}_{m}\right)+\frac{\eta_{m}}{b}\left[\Psi\left(x, b, \boldsymbol{\Omega}_{m}\right)-\Psi\left(x, 0, \boldsymbol{\Omega}_{m}\right)\right]+\sigma_{t} \Psi_{y}\left(x, \boldsymbol{\Omega}_{m}\right)= \\
& Q_{y}(x)+\frac{\sigma_{s}}{4} \sum_{k=1}^{M / 2} w_{k}\left[\Psi_{y}\left(x, \boldsymbol{\Omega}_{k}\right)+\Psi_{y}\left(x, \boldsymbol{\Omega}_{k+M / 2}\right)\right]
\end{aligned}
$$




$$
\begin{gathered}
-\mu_{m} \frac{d}{d x} \Psi_{y}\left(x, \boldsymbol{\Omega}_{m+M / 2}\right)+\frac{\eta_{m+M / 2}}{b}\left[\Psi\left(x, b, \boldsymbol{\Omega}_{m+M / 2}\right)-\Psi\left(x, 0, \boldsymbol{\Omega}_{m+M / 2}\right)\right]+ \\
\sigma_{t} \Psi_{y}\left(x, \boldsymbol{\Omega}_{m+M / 2}\right)=Q_{y}(x)+\frac{\sigma_{s}}{4} \sum_{k=1}^{M / 2} w_{k}\left[\Psi_{y}\left(x, \boldsymbol{\Omega}_{k}\right)+\Psi_{y}\left(x, \boldsymbol{\Omega}_{k+M / 2}\right)\right]
\end{gathered}
$$

para $m=1, \ldots, M / 2$, onde os momentos do fluxo angular são definidos como

$$
\Psi_{y}\left(x, \boldsymbol{\Omega}_{m}\right)=\frac{1}{b} \int_{0}^{b} \Psi\left(x, y, \boldsymbol{\Omega}_{m}\right) d y
$$

e a fonte

$$
Q_{y}(x)=\frac{1}{b} \int_{0}^{b} Q(x, y) d y .
$$

Analisando as condições de contorno do problema, verifica-se que os fluxos nas direções de incidência são nulos em $y=b$ e $y=0$, ou seja:

$$
\Psi\left(x, b, \boldsymbol{\Omega}_{m}\right)=0 \quad m=M / 4+1, \ldots, M / 2 \quad \text { e } \quad m=3 M / 4+1, \ldots, M
$$

$\mathrm{e}$

$$
\Psi\left(x, 0, \boldsymbol{\Omega}_{m}\right)=0 \quad m=1, \ldots, M / 4 \quad \text { e } \quad m=M / 2+1, \ldots, 3 M / 4 .
$$

Por sua vez, os fluxos emergentes nas fronteiras são desconhecidos. Estes fluxos serão, então, aproximados da forma

$$
\Psi\left(x, b, \boldsymbol{\Omega}_{m}\right) \approx \widehat{k}_{2} \Psi_{y}\left(x, \boldsymbol{\Omega}_{m}\right) \quad m=1, \ldots, M / 4 \quad \text { e } \quad m=M / 2+1, \ldots, 3 M / 4,
$$

e

$$
\Psi\left(x, 0, \boldsymbol{\Omega}_{m}\right) \approx \widehat{k}_{1} \Psi_{y}\left(x, \boldsymbol{\Omega}_{m}\right) \quad m=M / 4+1, \ldots, M / 2 \quad \text { e } \quad m=3 M / 4+1, \ldots, M
$$

afim de obter condições auxiliares para resolver o sistema de equações. Neste ponto, considera-se as constantes $\widehat{k}_{1}$ e $\widehat{k}_{2}$ arbitrárias.

Substituindo-se as eqs. (6) e (7) e as aproximações definidas nas eqs. (8) e (9) nas eqs. (2) e (3), obtém-se o seguinte conjunto de equações unidimensionais na direção $x$

$$
\begin{gathered}
\mu_{m} \frac{d}{d x} \Psi_{y}\left(x, \boldsymbol{\Omega}_{m}\right)+\left[\sigma_{t}+k_{2} \eta_{m}\right] \Psi_{y}\left(x, \boldsymbol{\Omega}_{m}\right)= \\
Q_{y}(x)+\frac{\sigma_{s}}{4} \sum_{k=1}^{M / 2} w_{k}\left[\Psi_{y}\left(x, \boldsymbol{\Omega}_{k}\right)+\Psi_{y}\left(x, \boldsymbol{\Omega}_{k+M / 2}\right)\right] \\
\mu_{m+M / 4} \frac{d}{d x} \Psi_{y}\left(x, \boldsymbol{\Omega}_{m+M / 4}\right)+\left[\sigma_{t}-k_{1} \eta_{m+M / 4}\right] \Psi_{y}\left(x, \boldsymbol{\Omega}_{m+M / 4}\right)= \\
Q_{y}(x)+\frac{\sigma_{s}}{4} \sum_{k=1}^{M / 2} w_{k}\left[\Psi_{y}\left(x, \boldsymbol{\Omega}_{k}\right)+\Psi_{y}\left(x, \boldsymbol{\Omega}_{k+M / 2}\right)\right] \\
-\mu_{m} \frac{d}{d x} \Psi_{y}\left(x, \boldsymbol{\Omega}_{m+M / 2}\right)+\left[\sigma_{t}+k_{2} \eta_{m+M / 2}\right] \Psi_{y}\left(x, \boldsymbol{\Omega}_{m+M / 2}\right)= \\
Q_{y}(x)+\frac{\sigma_{s}}{4} \sum_{k=1}^{M / 2} w_{k}\left[\Psi_{y}\left(x, \boldsymbol{\Omega}_{k}\right)+\Psi_{y}\left(x, \boldsymbol{\Omega}_{k+M / 2}\right)\right]
\end{gathered}
$$


$\mathrm{e}$

$$
\begin{gathered}
-\mu_{m+M / 4} \frac{d}{d x} \Psi_{y}\left(x, \boldsymbol{\Omega}_{m+3 M / 4}\right)+\left[\sigma_{t}-k_{1} \eta_{m+3 M / 4}\right] \Psi_{y}\left(x, \boldsymbol{\Omega}_{m+3 M / 4}\right)= \\
Q_{y}(x)+\frac{\sigma_{s}}{4} \sum_{k=1}^{M / 2} w_{k}\left[\Psi_{y}\left(x, \boldsymbol{\Omega}_{k}\right)+\Psi_{y}\left(x, \boldsymbol{\Omega}_{k+M / 2}\right)\right],
\end{gathered}
$$

para $m=1, \ldots, M / 4$ e $k_{1}=\widehat{k}_{1} / b$ e $k_{2}=\widehat{k}_{2} / b$.

Verifica-se que, utilizando esta abordagem, os termos derivados dos fluxos desconhecidos na fronteira não são introduzidos como modificações no termo fonte do problema original, permanecendo o problema derivado da direção $x$ desacoplado da direção $y$.

\section{Solução em ordenadas discretas}

A partir da equação de transporte bidimensional, conseguiu-se gerar um sistema de equações unidimensionais (10)-(13), possibilitando o uso do método ADO para sua resolução. As soluções homogêneas obtidas através deste método, são construídas em termos de autovalores e de autofunções. São propostas, então, soluções da forma

$$
\Psi_{y}\left(x, \boldsymbol{\Omega}_{m}\right)=\Phi\left(\nu, \boldsymbol{\Omega}_{m}\right) e^{-x / \nu} .
$$

De acordo com o trabalho anterior [10], é obtido via o método ADO, o seguinte problema de autovalores escrito na forma matricial $[\mathbf{D}-\mathbf{A}] \mathbf{U}=\lambda \mathbf{U}$, onde $\mathbf{D}$ e $\mathbf{A}$ são matrizes $M / 2 \times M / 2$ e $\operatorname{com} \lambda=1 / \nu^{2}$. Aqui, a matriz $\mathbf{D}$ é definida como

$$
\begin{array}{r}
\mathbf{D}=\operatorname{diag}\left\{\left(\frac{\sigma_{t}+k_{2} \eta_{1}}{\mu_{1}}\right)^{2}, \ldots,\left(\frac{\sigma_{t}+k_{2} \eta_{M / 4}}{\mu_{M / 4}}\right)^{2},\right. \\
\left.\left(\frac{\sigma_{t}-k_{1} \eta_{M / 4+1}}{\mu_{M / 4+1}}\right)^{2}, \ldots,\left(\frac{\sigma_{t}-k_{1} \eta_{M / 2}}{\mu_{M / 2}}\right)^{2}\right\}
\end{array}
$$

e os elementos de $\mathbf{A}$ são representados, para $j=1, \ldots, M / 2$, como

$$
\mathbf{A}(i, j)=\left\{\begin{array}{lll}
\frac{w_{j} \sigma_{s}\left[\sigma_{t}+k_{2} \eta_{i}\right]}{2 \mu_{i}^{2}}, & \text { para } & i=1, \ldots, M / 4, \\
\frac{w_{j} \sigma_{s}\left[\sigma_{t}-k_{1} \eta_{i}\right]}{2 \mu_{i}^{2}}, & \text { para } & i=M / 4+1, \ldots, M / 2,
\end{array}\right.
$$

para $i=1, \ldots, M / 2$. Resolvido o problema de autovalores $\left\{\lambda_{j}, \mathbf{U}_{j}\right\}$ e determinadas as constantes de separação $\nu_{j}$, definem-se as funções $\mathbf{V}$ de acordo com a referencia [10], e determina-se as autofunções que podem ser escritas, para $m=1, \ldots, M / 2$, na forma

$$
\Phi\left(\nu_{j}, \boldsymbol{\Omega}_{m}\right)=\frac{U\left(\nu, \boldsymbol{\Omega}_{m}\right)+V\left(\nu, \boldsymbol{\Omega}_{m}\right)}{2}
$$

e

$$
\Phi\left(\nu_{j}, \boldsymbol{\Omega}_{m+M / 2}\right)=\frac{U\left(\nu, \Omega_{m}\right)-V\left(\nu, \boldsymbol{\Omega}_{m}\right)}{2} .
$$

Como as constantes de separação aparecem aos pares $\pm \nu_{j}$, podemos escrever a solução do problema homogêneo da eq. (2) e (3), para $m=1, \ldots, M / 2$ na forma geral

$$
\Psi_{y}^{h}\left(x, \boldsymbol{\Omega}_{m}\right)=\sum_{j=1}^{M / 2} A_{j} \Phi\left(\nu_{j}, \boldsymbol{\Omega}_{m}\right) e^{-x / \nu_{j}}+B_{j} \Phi\left(\nu_{j}, \boldsymbol{\Omega}_{m+M / 2}\right) e^{-(a-x) / \nu_{j}}
$$

$\mathrm{e}$

$$
\Psi_{y}^{h}\left(x, \boldsymbol{\Omega}_{m+M / 2}\right)=\sum_{j=1}^{M / 2} A_{j} \Phi\left(\nu_{j}, \boldsymbol{\Omega}_{m+M / 2}\right) e^{-x / \nu_{j}}+B_{j} \Phi\left(\nu_{j}, \boldsymbol{\Omega}_{m}\right) e^{-(a-x) / \nu_{j}},
$$

onde os coeficientes $A_{j}$ e $B_{j}$ devem ser determinados. 


\subsection{Solução Particular}

Para escrever a solução geral da eqs. (2) e (3) seguiu-se o trabalho anterior [5], que faz uso das funções de Green e as soluções elementares do problema para construir a solução particular. Desta forma, a solução é proposta como

$$
\Psi_{y}^{p}\left(x, \boldsymbol{\Omega}_{m}\right)=\sum_{j=1}^{M / 2} C_{j}(x) \Phi\left(\nu_{j}, \boldsymbol{\Omega}_{m}\right)+D_{j}(x) \Phi\left(\nu_{j}, \boldsymbol{\Omega}_{m+M / 2}\right)
$$

$\mathrm{e}$

$$
\Psi_{y}^{p}\left(x, \boldsymbol{\Omega}_{m+M / 2}\right)=\sum_{j=1}^{M / 2} C_{j}(x) \Phi\left(\nu_{j}, \boldsymbol{\Omega}_{m+M / 2}\right)+D_{j}(x) \Phi\left(\nu_{j}, \boldsymbol{\Omega}_{m}\right),
$$

onde para o caso teste deste trabalho, diferentemente de [5], deriva-se explicitamente as seguintes expressões para $C_{j}(x)$ e $D_{j}(x)$,

$$
C_{j}(x)=\frac{1}{N\left(\nu_{j}\right)} \sum_{\alpha=1}^{M / 2} \nu_{j} \omega_{\alpha} \frac{b_{s}}{b}\left[\left[\Phi\left(\nu_{j}, \boldsymbol{\Omega}_{\alpha}\right)+\Phi\left(\nu_{j}, \boldsymbol{\Omega}_{\alpha+M / 2}\right)\right]\left(1-e^{-x / \nu_{j}}\right)\right]
$$

e

$$
D_{j}(x)=\frac{1}{N\left(\nu_{j}\right)} \sum_{\alpha=1}^{M / 2} \nu_{j} \omega_{\alpha} \frac{b_{s}}{b}\left[\left[\Phi\left(\nu_{j}, \boldsymbol{\Omega}_{\alpha+M / 2}\right)+\Phi\left(\nu_{j}, \boldsymbol{\Omega}_{\alpha}\right)\right]\left(1-e^{-\left(a_{s}-x\right) / \nu_{j}}\right)\right],
$$

para $x \in\left[0, a_{s}\right]$, ainda,

$$
C_{j}(x)=\frac{1}{N\left(\nu_{j}\right)} \sum_{\alpha=1}^{M / 2} \nu_{j} \omega_{\alpha} \frac{b_{s}}{b}\left[\left[\Phi\left(\nu_{j}, \boldsymbol{\Omega}_{\alpha}\right)+\Phi\left(\nu_{j}, \boldsymbol{\Omega}_{\alpha+M / 2}\right)\right]\left(e^{-\left(x-a_{s}\right) / \nu_{j}}-e^{-x / \nu_{j}}\right)\right]
$$

e

$$
D_{j}(x)=0
$$

para $a_{s}<x \leq a$, onde

$$
N\left(\nu_{j}\right)=\sum_{k=1}^{M / 2} \omega_{k} \mu_{k}\left[\Phi^{2}\left(\nu_{j}, \boldsymbol{\Omega}_{k}\right)-\Phi^{2}\left(\nu_{j}, \boldsymbol{\Omega}_{k+M / 2}\right)\right] .
$$

Objetivando validar os resultados numéricos obtidos em [10], considerou-se aqui, $Q\left(x, \boldsymbol{\Omega}_{\alpha}\right)=$ 1 , para $x \in\left[0, a_{S}\right]$. Definidas as soluções homogênea e particular para as eqs. (10)-(13) pode-se, agora, determinar as constantes $A_{j}$ e $B_{j}$. Considerando a versão integrada das condições de contorno do problema

$$
\Psi_{y}\left(0, \Omega_{m}\right)=0, \quad m=1, \ldots, M / 2
$$

e

$$
\Psi_{y}\left(a, \boldsymbol{\Omega}_{m}\right)=0, \quad m=M / 2+1, \ldots, M,
$$

e substituindo-as nas eqs. (19)-(22), obtém-se o seguinte sistema linear para $m=1, \ldots, M / 2$

$$
\sum_{k=1}^{M / 2} A_{k} \Phi\left(\nu_{k}, \boldsymbol{\Omega}_{m}\right)+B_{k} \Phi\left(\nu_{k}, \boldsymbol{\Omega}_{m+M / 2}\right) e^{-a / \nu_{k}}=-\Psi_{y}^{p}\left(0, \boldsymbol{\Omega}_{m}\right)
$$

e

$$
\sum_{k=1}^{M / 2} A_{k} \Phi\left(\nu_{k}, \boldsymbol{\Omega}_{m+M / 2}\right) e^{-a / \nu_{k}}+B_{k} \Phi\left(\nu_{k}, \boldsymbol{\Omega}_{m}\right)=-\Psi_{y}^{p}\left(a, \boldsymbol{\Omega}_{m+M / 2}\right) .
$$

A resolução deste sistema linear $M \times M$ torna a solução completamente estabelecida e podese, então, escrever a solução geral para o problema, como a soma da solução homogênea com a solução particular. 


\section{Considerações Finais}

Neste trabalho, buscando-se validar as soluções explícitas que vem sendo desenvolvidas para problemas bidimensionais de transporte através do método ADO conjuntamente com esquemas nodais, o caso teste tratado na referência [10] foi novamente abordado a partir de soluções diferenciadas no que diz respeito a solução particular do problema. Os resultados numéricos foram verificados em todos os dígitos, não sendo por isso aqui repetidos. A solução particular baseada no uso de funções de Green se torna mais abrangente, podendo incluir termos de fonte não constantes, e aqui, adicionalmente ao caso abordado na referência [5] foi mais uma vez verificada com sucesso.

\section{Referências}

[1] A.K. Al-Basheer, G.E. Sjoden, M. Ghita, Critical discretization issues in 3-D $S_{N}$ simulations relevant to dosimetry and medical physics, Nuclear Technology, 169 (2010) 252-262.

[2] Y.Y. Azmy, Comparison of three approximations to the linear-linear nodal transport method in weighted diamonddifference form, Nuclear Science and Engineering, 100 (1988) 190-200.

[3] A. Badruzzaman, An efficient algorithm for nodal-transport solutions in multidimensional geometry, Nuclear Science and Engineering, 89 (1985) 281-290.

[4] L.B. Barichello, C.E. Siewert, A discrete-ordinates solution for a non-grey model with complete frequency redistribution, Journal of Quantitative Spectroscopy and Radiative Transfer, 62 (1999) 665-675.

[5] L.B. Barichello, L.C. Cabrera, J.F.P. Filho, An analytical discrete ordinates solution for two dimensional problems based on nodal schemes, "In Proceedings of the International Nuclear Atlantic Conference - INAC2009", Rio de Janeiro, Brazil, 2009.

[6] L.B. Barichello, L.C. Cabrera, J.F.P. Filho, An analytical approach for a nodal scheme of two-dimensional neutron transport problems, Annals of Nuclear Energy, 38 (2011) 13101317.

[7] J.I. Duo, Y.Y. Azmy, L.T. Zikatanov, A posteriori error estimator and AMR for discrete ordinates nodal transport methods, Annals of Nuclear Energy, 36 (2009) 268-273.

[8] R.R. Gomes, R.C. Barros, Computational modeling of monoenergetic neutral particle inverse transport problems in slab geometry, American Institute of Physics, 1479 (2012) $2225-2228$.

[9] S.K. Loyalka, R.W. Tsai, A numerical method for solving the integral equation of neutron transport: II, Nuclear Science and Engineering, 58 (1975) 193-202.

[10] A. Tres, C. Becker, R.D. Cunha, L.B. Barichello, A deterministic approach for neutron transport in two-dimensional domains, "In Proceedings of the International Congress of Mechanical Engineering - COBEM", Ribeirão Preto, Brazil, 2013, aceito para publicação.

[11] M.M.R. Williams, Radiation transport in a light duct using a one-dimensional model, Physica Scripta, 76 (2007) 303-313.

[12] M.M.R. Williams, Exact solutions of the two-dimensioanl cell problem, Nuclear Science and Engineering, 173 (2012) 33-51. 